\title{
An Unusual Presentation of a Solid Pseudopapillary Tumor of the Pancreas Mimicking Adenocarcinoma
}

\author{
Hyung Ku Chon ${ }^{1,2}$, Keum Ha Choi ${ }^{3}$ and Tae Hyeon Kim \\ ${ }^{1}$ Department of Internal Medicine, ${ }^{2}$ Institute of Wonkwang Medical Science, ${ }^{3}$ Department of Pathology, Wonkwang University College of
} Medicine and Hospital, Iksan, Korea

Solid pseudopapillary tumors of the pancreas are rare and typically occur in young women. Compared with pancreatic adenocarcinoma, solid pseudopapillary tumors are characterized by notable indolent biological behavior associated with a favorable prognosis. Despite their large size, these tumors rarely metastasize. Even in cases of hepatic metastasis, most lesions are usually solitary in distribution and are amenable to resection. We report a case of a 55-year-old man with a small solid pseudopapillary tumor $(\leq 3$-cm diameter) mimicking a pancreatic adenocarcinoma, with multiple hepatic metastases. The diagnosis was confirmed by endoscopic ultrasoundguided fine-needle biopsy using a 22-G core needle. Unfortunately, rapid tumor progression led to patient mortality 5 months after diagnosis. To our knowledge, this is the first case report that describes a small solid pseudopapillary tumor of the pancreas with multiple hepatic metastasis and poor prognosis in a patient who was diagnosed with this condition at the time of initial diagnosis. Clin Endosc 2020;53:615-619

Key Words: Endoscopic ultrasound-guided fine-needle biopsy; Neoplasm metastasis; Pancreatic neoplasm

\section{INTRODUCTION}

A solid pseudopapillary tumor (SPT) of the pancreas is a rare tumor of low malignant potential, accounting for only $1 \%-3 \%$ of pancreatic neoplasms. ${ }^{1}$ SPTs commonly occur in young women and rarely metastasize, and the 5-year survival rate is estimated to be approximately $95 \%-97 \% .{ }^{2}$ A few SPTs show markedly aggressive behavior indicated by a tumor grade $\geq \mathrm{T} 3$, perineural and vascular invasion, and lymph node or distant metastasis. However, to date, no report has described a small SPT ( $<3-\mathrm{cm}$ diameter) associated with multiple hepatic metastases identified in a male patient at the time of initial diagnosis. Interestingly, tumor progression in our

Received: August 7, 2019 Revised: September 4, 2019

Accepted: September 6, 2019

Correspondence: Hyung Ku Chon

Department of Internal Medicine, Wonkwang University College of Medicine and Hospital, 895 Muwang-ro, Iksan 54538, Korea

Tel: +82-63-859-2564, Fax: +82-63-855-2025, E-mail: gipb2592@wku.ac.kr ORCID: http://orcid.org/0000-0002-6068-3849

(cc) This is an Open Access article distributed under the terms of the Creative Commons Attribution Non-Commercial License (http://creativecommons.org/ licenses/by-nc/3.0) which permits unrestricted non-commercial use, distribution, and reproduction in any medium, provided the original work is properly cited. patient was relatively rapid in contrast to the indolent tumor behavior that commonly characterizes SPTs.

\section{CASE REPORT}

A 55-year-old man without any underlying disease was admitted to our hospital for evaluation of a small pancreatic mass and multiple hepatic lesions, which were incidentally detected during ultrasonography. He was asymptomatic and denied a history of smoking or heavy alcohol consumption. Laboratory investigations revealed his hepatic parameters and tumor markers were within normal limits. Abdomen computed tomography $(\mathrm{CT})$ revealed a $2.5-\mathrm{cm}$ low attenuating mass in the pancreatic head and a less-enhancing lesion in the hepatic lobe (Fig. 1A, B). Magnetic resonance imaging, using axial dynamic fat-saturated T1-weighted images obtained in the arterial, portal, and delayed phases, showed an ill-defined mass, $2.5 \mathrm{~cm}$ in diameter, with persistent delayed enhancement in the pancreatic head along with multiple low-intensity signals in both hepatic lobes (Fig. 1C, D) without any evidence of biliary and/or pancreatic duct dilatation 
or pancreatic parenchymal atrophy. Endoscopic ultrasound (EUS) examination revealed a mass of ill-defined mixed echogenicity, measuring approximately $2.6 \mathrm{~cm}$, with calcifications in the pancreatic head and multiple irregular margins with low, dense lesions in both hepatic lobes (Fig. 1E, F). EUS-guided fine-needle biopsy (EUS-FNB) was performed using a 22-G core needle (EchoTip ProCore ${ }^{\circledR}$ HD Ultrasound Biopsy
Needle; Wilson-Cook Medical Inc., Bloomington, IN, USA). Histological examination of the biopsy specimen revealed scattered monotonous round cells. Tumor cells were uniform and formed delicate papillary fronds with hyalinized stroma (Fig. 2A). Immunohistochemical (IHC) examination revealed tumor cells that showed positive results with beta-catenin (Fig. 2B), alpha-1-antichymotrypsin, and CD 56 stains and negative
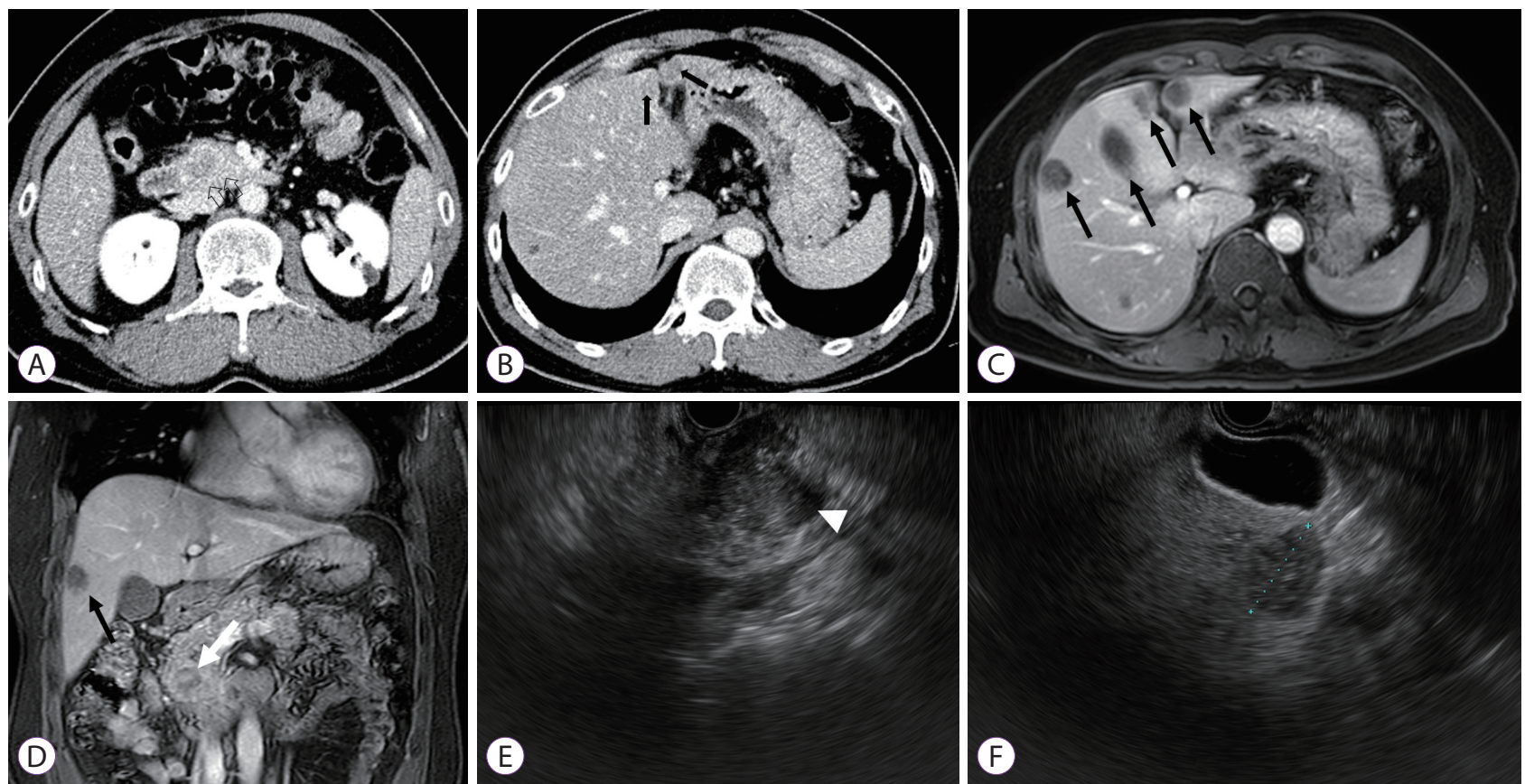

Fig. 1. Abdomen computed tomography scan showing (A) a $2.5 \mathrm{~cm}$ low attenuating mass (black open arrow) in the pancreatic head and (B) a less-enhancing lesion in the hepatic lobe (black arrows). Magnetic resonance imaging scans (arterial phase of an axial T-1 weighted image) showing ill-defined low signal intensity in the pancreatic head and (C) multiple hepatic lesions (black arrows). (D) Delayed phase of a coronal T-1 weighted image showing heterogenous echogenic lesions (white arrow) in the pancreatic head without main pancreatic duct dilatation or pancreatic parenchymal atrophy. An ill-defined low signal intensity (black arrow) is observed in the right hepatic lobe. Endoscopic ultrasound showing $(E)$ an ill-defined mixed echogenic mass in the pancreatic head and $(F)$ irregular margins with low, dense lesions in the right hepatic lobes.
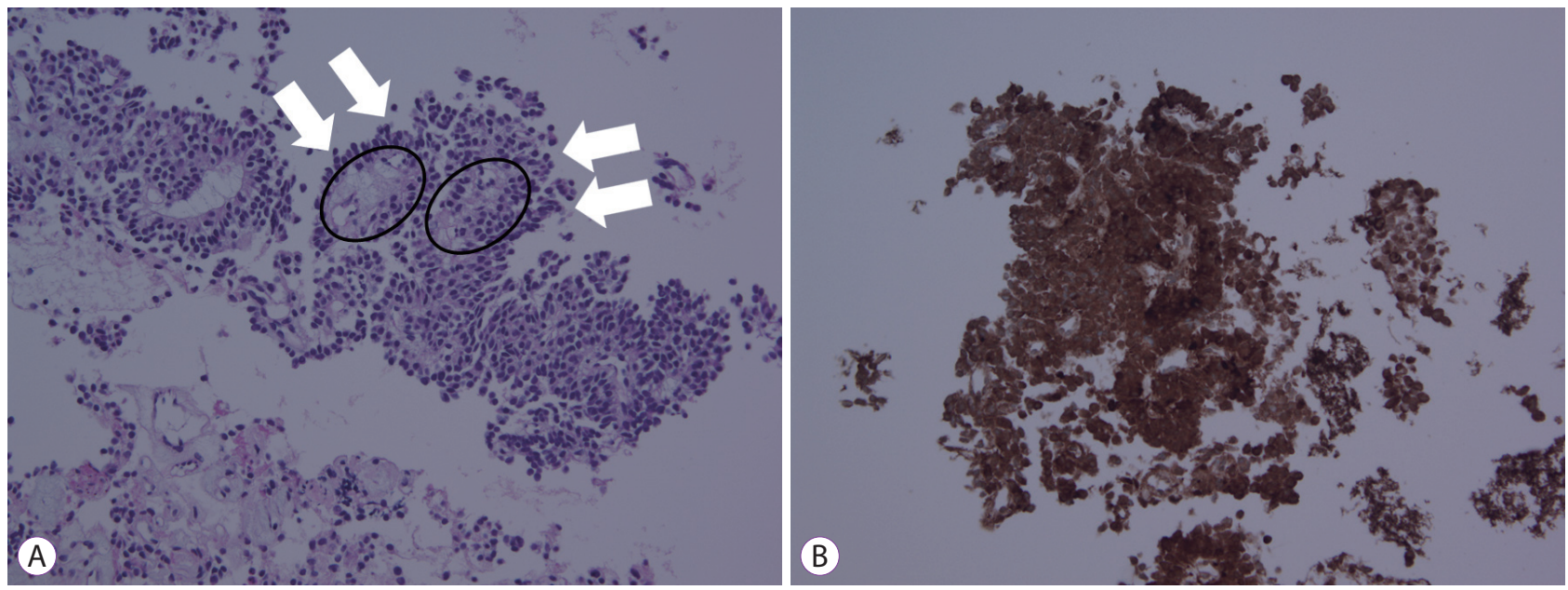

Fig. 2. Histopathological examination of a biopsy specimen showing (A) monomorphic cells with delicate papillary fronds (white arrows) without a fibrovascular core (black circles; hematoxylin \& eosin stain, $\times 200$ ). (B) Neoplastic cells are diffusely and strongly stained with beta-catenin (immunohistochemical staining, $\times 200$ ). 
results with synaptophysin and chromogranin A stains. The Ki-67 expression rate was $8 \%$. Based on the findings of histopathological and radiological examination, the patient was diagnosed with a pancreatic SPT with multiple hepatic metas-

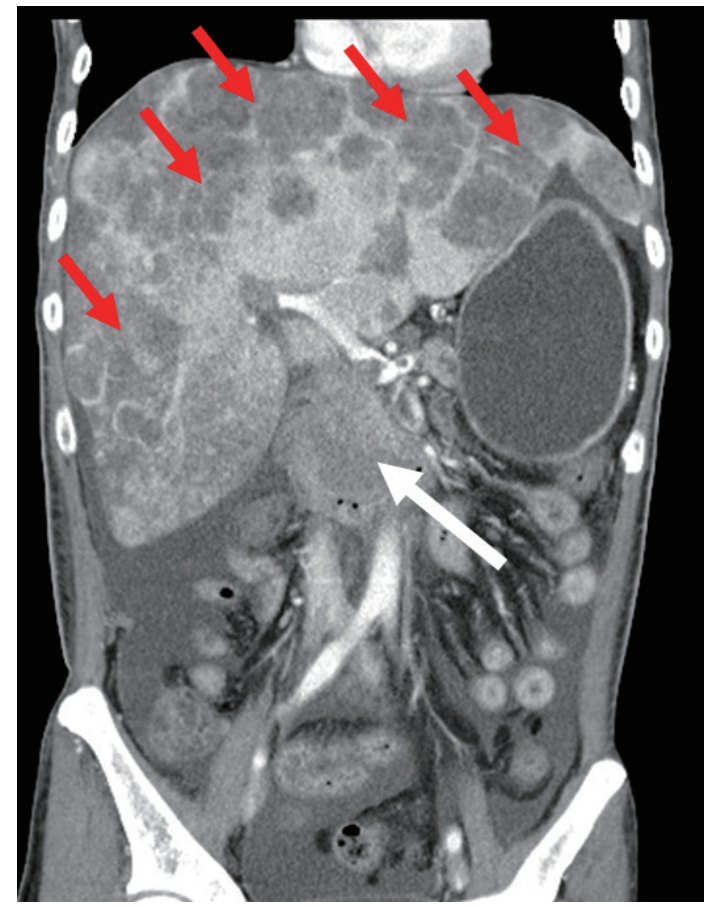

Fig. 3. Abdominal computed tomography scan showing an enlarged pancreatic mass (white arrow), multiple hepatic masses (red arrows), and massive ascites. tases. Unfortunately, the patient refused any other treatment and died 5 months later, secondary to tumor progression (Fig. 3).

\section{DISCUSSION}

SPTs of the pancreas are rare tumors with low malignant potential that show good prognosis after complete resection. Previous studies have reported a male:female ratio of 1:9.5, with a median age of 21.97 years and a mean tumor diameter of $6.08 \mathrm{~cm} .{ }^{3}$ SPTs typically occur in young women and present as large tumors. Notably, $<10 \%$ of patients with SPTs reported in the literature were men, with these lesions presenting a diagnostic challenge in those cases. Moreover, men with SPT tend to show late-onset disease and greater tumor aggressiveness than women. ${ }^{4}$

The typical CT and magnetic resonance imaging features of SPT include a large well-encapculated mass with a mixture of solid and cystic components accompanied by hemorrhage. The EUS findings of SPT show a well-defined homogenous, hypoechic mass with a hyperechic rim. In the present case, there was no surrounding capsule or cystic component with hemorrhage unlike typical SPT. Reportedly, small SPTs $(<3-\mathrm{cm}$ diameter) present as exclusively solid lesions without cystic components and calcification. ${ }^{5}$ Therefore, small SPTs may mimic pancreatic ductal adenocarcinoma (PDAC), although several radiological features of SPTs distinguish them from PDAC. ${ }^{5}$ Small SPTs show no upstream pancreatic duct dila-

Table 1. Summary of Solid Pseudopapillary Tumor with Hepatic Metastasis from Literature Review

\begin{tabular}{lccl}
\hline Study & Age/Sex & Size of tumor $(\mathbf{c m})$ & \multicolumn{1}{c}{ Detection of liver metastasis } \\
\hline Watanabe et al. $(2017)^{6}$ & $49 / \mathrm{F}$ & 11 & 3 mo after surgery \\
\hline Sperti et al. $(2008)^{7}$ & $49 / \mathrm{F}$ & 10 & 32 mo after surgery \\
\hline Tang et al. $(2005)^{8}$ & $45 / \mathrm{F}$ & 9 & 3 mo after surgery \\
& $33 / \mathrm{F}$ & 25 & Initial diagnosis \\
\hline Estrella et al. $(2014)^{1}$ & $11 / \mathrm{F}$ & 9.5 & Initial diagnosis \\
& $14 / \mathrm{F}$ & 10 & Initial diagnosis \\
& $35 / \mathrm{F}$ & 12 & 64 mo after surgery \\
& $61 / \mathrm{F}$ & 11 & 40 mo after surgery \\
Takahashi et al. $(2005)^{9}$ & $50 / \mathrm{F}$ & 13 & 78 mo after surgery \\
Wang et al. $(2014)^{10}$ & $41 / \mathrm{F}$ & 12 & Initial diagnosis \\
& $50 / \mathrm{F}$ & 6 & 72 mo after surgery \\
& $72 / \mathrm{F}$ & 4.1 & 72 mo after surgery \\
\hline Gomez et al. $(2012)^{11}$ & $20 / \mathrm{F}$ & 10 & 48 mo after surgery \\
\hline Present case & $22 / \mathrm{F}$ & 15 & Initial diagnosis \\
\hline
\end{tabular}


tation, pancreatic parenchymal atrophy, and metastasis to the intra-abdominal solid organs at the time of initial diagnosis. In contrast to the known indolent behavior of typical SPTs and despite its small size, the tumor metastasized to both hepatic lobes in our patient. According to a recent meta-analysis, the tumor size of SPTs with aggressive behavior was $9.75 \pm 4.77 \mathrm{~cm}$. To date, there have been no reported case of liver metastasis in SPTs $<3 \mathrm{~cm}$ in diameter (Table 1$)^{1,6-11}$ In consideration of the high female predilection of SPTs, the occurrence of this tumor in our patient was unusual; therefore, it was not initially considered in the differential diagnosis in this case.

Accurate preoperative diagnosis of an SPT is challenging. Reportedly, EUS-FNB using a core needle is a useful radiological diagnostic modality to evaluate indeterminate pancreatic lesions. ${ }^{12}$ Acquisition of core biopsy specimens followed by IHC staining and evaluation of the preserved tissue architecture may aid in diagnosis, as observed in our patient. Currently, most SPTs are diagnosed based on their gross and microscopic appearance. IHC staining is a useful technique for SPTs that are difficult to diagnose morphologically. With regards to IHC assays, positive results with the beta-catenin stain and negative results with neuroendocrine markers, such as chromogranin A or synaptophysin, are important indicators that distinguish SPTs from pancreatic neuroendocrine tumors. ${ }^{13}$

SPTs typically show indolent behavior and are associated with long survival rates (even in patients with disseminated disease) secondary to slow tumor doubling time (765 days). ${ }^{14}$ Unfortunately, rapid tumor progression led to our patient's mortality 5 months after being diagnosed with this tumor. Aggressive behavior of SPTs may be attributed to the relatively high Ki-67 index observed in histopathological examination. Several studies focusing on cell cycle analysis have reported the utility of Ki-67 expression as a cell proliferation marker in many different types of cancer. The percentage of tumor cells that show positive Ki-67 expression (determined by IHC analysis) is associated with patient outcomes, and a high Ki67 index is usually associated with poor prognosis in patients with lung, bladder, breast, and cervical cancer; and lymphoma. ${ }^{15}$ The Ki-67 expression rate was $8 \%$ in our patient. There is a lack of consensus regarding the utility of the Ki-67 index as a prognostic marker for SPT; however, Yang et al. ${ }^{16}$ reported that a $\mathrm{Ki}-67$ index $>4 \%$ may indicate poor prognosis in patients with SPT.

Surgery is the treatment of choice for SPTs, and metastasectomy should also be considered if possible. ${ }^{10,17}$ In patients with unresectable SPT, there is no consensus on treatment. Maffuz et al. reported an unresectable SPT treated effectively by surgical resection after preoperative chemotherapy with fluorouracil and radiation. ${ }^{18}$ Morikawa et al. showed the efficacy of paclitaxel chemotherapy in a patient with recurrent liver and lymph node metastasis, 3 months after primary SPT resection. ${ }^{19}$ However, several studies did not show any benefits of chemotherapy in patients with unresectable SPT. ${ }^{18-20}$ Therefore, the role of chemotherapy and/or radiotherapy in adjuvant, neo-adjuvant, or metastatic settings has not been conclusively established. ${ }^{19}$ Surgery was contraindicated in our patient owing to multiple metastases in both hepatic lobes. Chemotherapy was considered an alternative treatment strategy; however, the patient refused all treatment.

According to our experience, when clinicians encounter patients with a small pancreatic mass and multiple hepatic lesions without pancreatic duct dilatation or parenchymal atrophy, a high index of suspicion of SPT should be considered during the differential diagnosis, regardless of gender. EUSFNB should be considered as a diagnostic modality for the evaluation of unusual pancreatic masses.

Conflicts of Interest

The authors have no financial conflicts of interest.

Acknowledgements

This study was supported by the Institute of Wonkwang Medical Science in 2019.

Author Contributions

Conceptualization: Hyung Ku Chon

Data curation: $\mathrm{HKC}$

Formal analysis: HKC, Tae Hyeon Kim

Funding acquisition: $\mathrm{HKC}$

Resources: HKC, Keum Ha Choi

Supervision: HKC

Validation: HKC

Writing-original draft: $\mathrm{HKC}$

Writing-review\&editing: KHC, THK

ORCID

Keum Ha Choi: https://orcid.org/0000-0001-8617-4869

Tae Hyeon Kim: https://orcid.org/0000-0002-9723-2136

\section{REFERENCES}

1. Estrella JS, Li L, Rashid A, et al. Solid pseudopapillary neoplasm of the pancreas: clinicopathologic and survival analyses of 64 cases from a single institution. Am J Surg Pathol 2014;38:147-157.

2. Kosmahl M, Seada LS, Jänig U, Harms D, Klöppel G. Solid-pseudopapillary tumor of the pancreas: its origin revisited. Virchows Arch 2000;436:473-480.

3. Klimstra DS, Wenig BM, Heffess CS. Solid-pseudopapillary tumor of the pancreas: a typically cystic carcinoma of low malignant potential. Semin Diagn Pathol 2000;17:66-80.

4. Machado MC, Machado MA, Bacchella T, Jukemura J, Almeida JL, Cunha JE. Solid pseudopapillary neoplasm of the pancreas: distinct patterns of onset, diagnosis, and prognosis for male versus female patients. Surgery 2008;143:29-34.

5. Baek JH, Lee JM, Kim SH, et al. Small ( $<\mathrm{or}=3 \mathrm{~cm}$ ) solid pseudopapillary tumors of the pancreas at multiphasic multidetector CT. Radiology 
2010;257:97-106

6. Watanabe Y, Okamoto K, Okada K, Aikawa M, Koyama I, Yamaguchi $\mathrm{H}$. A case of aggressive solid pseudopapillary neoplasm: comparison of clinical and pathologic features with non-aggressive cases. Pathol Int 2017;67:202-207.

7. Sperti C, Berselli M, Pasquali C, Pastorelli D, Pedrazzoli S. Aggressive behaviour of solid-pseudopapillary tumor of the pancreas in adults: a case report and review of the literature. World J Gastroenterol 2008;14:960-965.

8. Tang LH, Aydin H, Brennan MF, Klimstra DS. Clinically aggressive solid pseudopapillary tumors of the pancreas: a report of two cases with components of undifferentiated carcinoma and a comparative clinicopathologic analysis of 34 conventional cases. Am J Surg Pathol 2005;29:512-519.

9. Takahashi Y, Fukusato T, Aita K, et al. Solid pseudopapillary tumor of the pancreas with metastases to the lung and liver. Pathol Int 2005;55:792-796.

10. Wang WB, Zhang TP, Sun MQ, Peng Z, Chen G, Zhao YP. Solid pseudopapillary tumor of the pancreas with liver metastasis: clinical features and management. Eur J Surg Oncol 2014;40:1572-1577.

11. Gomez P, Yorke R, Ayala AG, Ro JY. Solid-pseudopapillary neoplasm of pancreas with long delayed liver metastasis. Ann Diagn Pathol 2012;16:380-384.

12. Kim EY. Role of repeated endoscopic ultrasound-guided fine needle aspiration for inconclusive initial cytology result. Clin Endosc
2013;46:540-542.

13. Ohara Y, Oda T, Hashimoto S, et al. Pancreatic neuroendocrine tumor and solid-pseudopapillary neoplasm: key immunohistochemical profiles for differential diagnosis. World J Gastroenterol 2016;22:8596-8604.

14. Kato T, Egawa N, Kamisawa T, et al. A case of solid pseudopapillary neoplasm of the pancreas and tumor doubling time. Pancreatology 2002;2:495-498

15. Menon SS, Guruvayoorappan C, Sakthivel KM, Rasmi RR. Ki-67 protein as a tumour proliferation marker. Clin Chim Acta 2019;491:39-45.

16. Yang F, Yu X, Bao Y, Du Z, Jin C, Fu D. Prognostic value of Ki-67 in solid pseudopapillary tumor of the pancreas: Huashan experience and systematic review of the literature. Surgery 2016;159:1023-1031.

17. Fried P, Cooper J, Balthazar E, Fazzini E, Newall J. A role for radiotherapy in the treatment of solid and papillary neoplasms of the pancreas. Cancer 1985;56:2783-2785.

18. Maffuz A, Bustamante Fde T, Silva JA, Torres-Vargas S. Preoperative gemcitabine for unresectable, solid pseudopapillary tumour of the pancreas. Lancet Oncol 2005;6:185-186.

19. Morikawa T, Onogawa T, Maeda S, et al. Solid pseudopapillary neoplasms of the pancreas: an 18-year experience at a single Japanese institution. Surg Today 2013;43:26-32.

20. Kang CM, Kim KS, Choi JS, Kim H, Lee WJ, Kim BR. Solid pseudopapillary tumor of the pancreas suggesting malignant potential. Pancreas 2006;32:276-280. 